УДК 338.012

$10.17213 / 2075-2067-2021-1-158-166$

\title{
УПРАВЛЕНИЕ БИЗНЕС-ПРОЦЕССАМИ КОМПАНИИ ПРИ РЕАЛИЗАЦИИ ЦИФРОВОЙ ТРАНСФОРМАЦИИ ${ }^{1}$
}

\author{
(C) 2021 г. О. П. Овчинникова , М. М. Харламов **
}

"Российский университет дружбы народов, г. Москва, Россия

"* Научно-исследовательский испытательный Центр имени Ю. А. Гагарина, Московская обл., Звездный городок, Россия

Целью исследования является обоснование механизмов управления бизнес-процессами компании в условиях осуществления циифровой трансформациии.

Методологическую базу исследования составляют труды российских ученых (В. И. Ананьина, К.В. Зимина, М. И. Лугачева, Р. Д. Гимранова, А. И. Лькова, А. В. Батищева и др.), которые рассматривают подходык к реализации бизнес-процессов в современных условиях, в том числе в рамках цифррового предприятия. Кроме того, в основу исследования были положены труды зарубежных ученых, рассматривающих вопросы реализации стратегии ичифровой трансформации (F. Holotiuk, D. Beimborn, A. Singh, T. Hess, C. Matt и дp.), а также вопросы реализачии ВPM - business process management (M. Rosemann, J. vom Brocke, F. Imgrund, M. Fischer, C. Janiesch, A. Winkelmann u дp.).

Результаты исследования. В ходе исследования авторами были предложены рекомендации, которые следует учитывать при реализации ВРМ в условиях ичифровой трансформации, в особенности в рамках стратегии ичифрового развития предприятия (цифровая стратегия, скорость изменений, иифровая экспертиза, новые технологии, командная работа и коммуникаичии, прозрачность).

Перспективу исследования составляет дальнейшее развитие наработок авторов в области использования менеджмента бизнес-процессами при реализачии стратегии развития предприятия в циирровой среде.

Ключевые слова: циифровая трансформаџия; управление бизнес-прочессами; технологические решения; циифровая экспертиза.

\section{MANAGEMENT OF THE COMPANY'S BUSINESS PROCESSES IN THE IMPLEMENTATION OF DIGITAL TRANSFORMATION}

\section{(C) 2021 O. P. Ovchinnikova*, M. M. Kharlamov**}

\section{"Peoples' Friendship University of Russia, Moscow, Russia}

${ }^{* *} Y$ u. A. Gagarin Research \& Test Cosmonaut Training Center, Moscow reg., Star city, Russia

The aim of the study is to substantiate the mechanisms for managing the company's business processes in the context of digital transformation.

The methodological basis of the research is the works of Russian (V.I. Ananin, K. V. Zimin, M. I. Lugachev, R. D. Gimranov, A. I. Lykova, A. V. Batishchev, etc.), which consider the approaches

1 Исследование выполнено при финансовой поддержке РФФИ в рамках научного проекта №20-010-00257. 
to the implementation of business processes in modern conditions, including within the digital enterprise. In addition, the study was based on the works of foreign scientists considering the implementation of the digital transformation strategy (F. Holotiuk, D. Beimborn, A. Singh, T. Hess, $C$. Matt and others), as well as the implementation of BPM - business process managemen (M. Rosemann, J. vom Brocke, F. Imgrund, M. Fischer, C. Janiesch, A. Winkelmann et al.).

Research results. In the course of the study, the authors proposed recommendations that should be taken into account when implementing BPM in the context of digital transformation, especially as part of an enterprise digital development strategy (digital strategy, speed of change, digital expertise, new technologies, teamwork and communications, transparency).

The prospect of the study is the further development of the authors' developments in the field of using business process management in the implementation of the enterprise development strategy in the digital environment.

Key words: digital transformation; business process management; technological solutions; digital expertise.

Введение. Цифровая трансформация является в настоящее время наиболее востребованным направлением исследований. Даже ярые противники цифровизации понимают, что идет смена технологических формаций, замена большинства аналоговых бизнеспроцессов на цифровые. Многие процессы полностью переходят в цифровой формат, возникают новые конфигурации бизнеспроцессов, новые форматы коммуникаций, новые технологические решения. Организационные структуры становятся более гибкими, адаптируясь под изменчивость внешней среды. В связи с этим актуализируются вопросы, связанные с формированием стратегии цифровой трансформации в компаниях, с изменением управленческой парадигмы применительно к цифровым бизнес-процессам.

Обзор литературы. Цифровая трансформация изменяет не только отдельные отрасли и предприятия, но и общество в целом. Этот процесс поддерживается использованием целого пула новых технологических решений, которые достаточно быстро становятся массово используемыми (мобильные, облачные технологии, технологии искусственного интеллекта и т.п.) [12]. Вместе с тем цифровая трансформация и повсеместное внедрение цифровых технологий воспринимается как серьезная угроза традиционным бизнес-процессам и организационной структуре $[9,19]$. Однако большинство компаний понимает, что цифровая трансформация - это требование времени, подразумевая под цифровой трансформацией автоматизацию всех процессов [2], внедрение программного обеспечения в деятельность хозяйственных структур [16] и т.п. В таких компаниях возникает фрагментарная цифровизация [6], которая при возникновении стрессовых ситуаций (например, пандемии) не в состоянии обеспечить полноценную деятельность предприятия.

Следовательно, реализация стратегии цифровой трансформации является для компании точкой опоры для повышения собственной конкурентоспособности во внешней среде. Данная стратегия подразумевает создание гибких организационных структур, которые быстро адаптируются под изменение внешней среды [1], а также ориентацию на наиболее полное удовлетворение потребностей клиентов и оптимизацию бизнеспроцессов [20]. В основе структурных изменений в компаниях, в том числе в рамках повсеместного внедрения информационных технологий, по мнению ряда исследователей, лежат технологии управления бизнеспроцессами организации (BPM - business process management) $[3,5]$.

В работе [18] выделены шесть основных элементов структуры ВРМ: стратегическое планирование, руководство, информационные технологии, персонал, метод и культура. Следует отметить, что в последнее время во многих работах технологии ВРМ рассматриваются как первоначальный этап цифровой трансформации в организациях [13]. 
Вместе с тем цифровую трансформацию часто идентифицируют с автоматизацией всех процессов в организации, в том числе и бизнес-процессов $[10,14,15]$. Такой подход обеспечил информационным технологиям ключевую роль в реализации стратегий компаний по повышению собственной конкурентоспособности на рынке [17].

Цифровые технологии способны объединять людей, находящихся в различных географических локациях, в режиме реального времени, позволяют генерировать и анализировать большие объемы информации, изменяя в конечном итоге организационные и социальные ландшафты [11]. Это и является основой цифровой трансформации на макро- и микроуровне.

Теоретическая рамка исследования. Стратегия цифровой трансформации компании предполагает, что повышение конкурентоспособности компании обеспечивается за счет оптимизации бизнес-процессов и по- вышения производительности труда за счет широкомасштабного использования цифровых технологий.

Соответственно, цифровая трансформация требует большей прозрачности процессов и механизмов принятия решений, что обеспечивается за счет распределения задач между членами команды и перехода к более гибкой, адаптивной структуре управления. Жесткая централизация управления сменяется плоской структурой с расширенными функциональными полномочиями и ответственностью подразделений.

В деятельность компаний все больше внедряются информационные технологии для анализа процессов, их моделирования и реализации. Подразделения самостоятельно решают, какие организационные изменения они могут проводить, как оптимизировать бизнес-процессы, как стимулировать персонал и т.п. [7].

В работе [8] авторы выделили ряд требований к цифровой трансформации, которые,

\section{Требования к цифровой трансформации ${ }^{2}$}

Таблица 1

\begin{tabular}{|l|l|}
\hline \multicolumn{1}{|c|}{ Требование } & \multicolumn{1}{c|}{ Содержание } \\
\hline Стратегия & $\begin{array}{l}\text { Стратегия цифровой трансформации определяет цели, задачи, па- } \\
\text { раметры цифровой трансформации, а также учитывает управлен- } \\
\text { ческие инновации и оптимизацию бизнес-процессов }\end{array}$ \\
\hline Скорость & $\begin{array}{l}\text { Учитывая высокую динамику внешней среды, компании внедряют } \\
\text { гибкие, адаптивные методы управления и гибкие организационные } \\
\text { структуры, позволяющие быстро реагировать на изменение вне- } \\
\text { шних условий }\end{array}$ \\
\hline Цифровая экспертиза & $\begin{array}{l}\text { Необходимость постоянного обучения, овладения новыми компе- } \\
\text { тенциям для решения более сложных задач }\end{array}$ \\
\hline Новые технологии & $\begin{array}{l}\text { Активное внедрение стандартизации и автоматизации в бизнес- } \\
\text { процессы организации }\end{array}$ \\
\hline $\begin{array}{l}\text { Командная работа и } \\
\text { коммуникации }\end{array}$ & $\begin{array}{l}\text { При цифровой трансформации возникает возможность и необходи- } \\
\text { мость перевода ряда процессов в дистанционный формат. Следо- } \\
\text { вательно, необходима организация эффективного взаимодействия } \\
\text { участников бизнес-процесса в удаленном доступе }\end{array}$ \\
\hline Прозрачность & $\begin{array}{l}\text { Для обеспечения устойчивости преобразований компании обеспе- } \\
\text { чивают прозрачность деятельности, которая способствует повыше- } \\
\text { нию доверия к компании со стороны стейкхолдеров }\end{array}$ \\
\hline
\end{tabular}

2 Источник: составлено авторами по Fischera M., Imgrunda F., Janiescha C., Winkelmanna A. (2020) Strategy archetypes for digital transformation: Defining meta objectives using business process management, Information \& Management. URL: https://doi.org/10.1016/j.im.2019.103262. 
учитывая изменения бизнес-процессов при цифровой трансформации, можно сформулировать следующим образом (таблица 1).

ВРМ напрямую или опосредованно удовлетворяет требованиям цифровой трансформации, указанным в таблице 1. Например, для обеспечения эффективной коммуникации в цифровой среде необходимо выстроить бизнес-процессы таким образом, чтобы все участники процесса могли осуществлять взаимодействие в электронной корпоративной среде, обеспечивающей быстрый и эффективный обмен информацией и знаниями. Взаимодействие членов команды в электронной среде способствует высокой скорости реагирования на возникающие проблемы, что в свою очередь способствует повышению качества обслуживания и удовлетворенности клиентов.

Вместе с тем цифровая трансформация ориентирована в конечном счете на повышение качества жизни населения за счет обеспечения доступа к широкому спектру продуктов и услуг с помощью цифровых технологий. Следовательно, ВРМ помимо классической ориентации на снижение затрат и повышение конкурентоспособности должна также учитывать необходимость повышения клиентоориентированности компании, что находит отражение в стратегии цифровой трансформации.

Высокая динамика рынка требует от компаний реализации ВРМ как последовательности взаимосвязанных инициатив, которые различаются по объему, структуре и целям и учитывают постоянные изменения внешней среды. Результаты ВРМ используются для улучшения организационной деятельности предприятия.

Важным моментом в рамках ВРМ является заинтересованность сотрудников в реализации изменений, в улучшении процессов и фиксации этих улучшений. Компании, которые поддерживают инициативы сотрудников по улучшению бизнес-процессов, опираются на формализацию навыков, технологий, документов, обеспечение доступа всех заинтересованных сторон к результатам улучшений, формируя в организации определенную динамичную знаниевую среду, которая является составной частью цифровой трансформации. Доступ к лучшим практикам в компании позволяет экономить ресурсы на разработку уп- равленческих и технологических решений, позволяющих улучшать бизнес-процессы.

Управление бизнес-процессами в компаниях опирается на определенные технологии моделирования бизнес-процессов, напримep, BPMN (Business Process Management Notation). По сути, это язык моделирования бизнес-процессов, позволяющий выстраивать бизнес-процесс и регламентировать его для всех участников, а в дальнейшем проводить автоматизацию бизнес-процессов в соответствии со схемой бизнес-процесса. Большинство компаний внедряют различные системы управления для мониторинга организационных изменений и используют репозитории для формирования итоговых моделей процессов [4].

При реализации цифровой трансформации для компаний важно понимать, что персонал является основой всех процессов. Следовательно, усилия компании должны быть направлены на повышение квалификации своих сотрудников, а также на обеспечение открытости бизнес-процессов и включенности сотрудников.

Большинство компаний предлагают учебные курсы для обучения своих сотрудников методам ВРМ и ориентации процессов на цифровую трансформацию. В ряде случаев компании создают справочники, которые подключаются к среде ВРМ. Система управления знаниями, создаваемая в компании, способствует повышению осведомленности сотрудников о бизнес-процессах путем проведения встреч, на которых все участники ВРМ обмениваются опытом, а также путем образовательных программ, что позволяет всем сотрудникам «видеть картину в целом», понимать бизнес-процессы и направлять свои усилия на их оптимизацию.

Результаты исследования. При реализации цифровой трансформации компании первоначально должны проанализировать бизнес-среду, определить свои приоритеты в бизнесе, а также провести оптимизацию бизнес-процессов. При этом необходимо понимать, что следует также учитывать организационные и экологические (в современных условиях — и эпидемиологические) условия, которые в значительной степени влияют на реализацию проектов. 
Представляется возможным выделить некоторые рекомендации при реализации ВРМ, которые соотносятся с требованиями к цифровой трансформации.

1. Цифровая стратегия. Определение целей стратегии должно основываться на выделенных приоритетах. ВРМ помогает достигать различных целей, вместе с тем для реализации эффективной стратегии следует сосредоточиться на определенных приоритетах, например, повышении адаптивности организации. Каждая масштабная цель должна иметь собственную стратегию реализации, следовательно, необходимо выстраивать приоритетность проектов и задач при реализации стратегии.

Кроме того, необходимо создать адекватные механизмы реализации стратегии. Масштабность цели и реализуемых проектов может быть недостижимыми вследствие неясных механизмов реализации, нечеткого распределения функций между исполнителями и негибкости процессов.

2. Скорость изменений. В современных условиях скорость принятия решений и гибкость бизнес-процессов является условиями обеспечения конкурентных преимуществ. Управленческие решения должны приниматься быстро в зависимости от изменения текущей ситуации и в кратчайшие сроки доводиться до исполнителей. В связи с этим необходимы отлаженные процессы передачи информации к руководству и каналы взаимодействия в рамках бизнес-процессов. Вместе с тем все участники бизнес-процесса должны понимать, почему были приняты данные решения и что необходимо делать для их реализации. В данном случае оптимальной видится архитектура процессов, которая включает в себя стратегический уровень, бизнесуровень и операционный уровень. Данная архитектура позволит увеличить скорость передачи информации по уровням, т.к. незначительная для высшего уровня информация может обрабатываться и анализироваться на более низком уровне, на котором и будут приниматься оперативные решения в рамках полномочий данного уровня.

3. Цифровая экспертиза. Для эффективной реализации цифровой трансформации компании необходимо иметь подготовленный персонал. Следует отметить, что речь идет не только об уровне образования кадров, но также и об уровне их осведомленности в рамках бизнес-процессов, их понимания концепции развития компании в целом или проекта в частности. Обучение персонала играет существенную роль в успехе цифровой трансформации.

4. Новые технологии. Основным преимуществом использования ВРМ при реализации цифровой трансформации является возможность выявления имеющихся компетенций (лучшие практики). Использование этих компетенций в образовательной среде организации позволяет совершенствовать организационные процедуры. Использование новых технологических решений повышает эффективность ВРМ и цифровой трансформации компании в целом. Повышение эффективности может быть достигнуто за счет интегрированной ИТ-инфраструктуры, которая позволяет использовать результаты ВРМ в деятельности компании (например, в виде общего репозитория, полностью интегрированного с другими технологическими решениями) [8].

5. Командная работа и коммуникации. В настоящее время компании, как правило, реализуют несколько проектов одновременно. Данные проекты имеют нередко разные цели и разные механизмы реализации. Вместе с тем данные проекты могут реализовываться людьми, которые входят в команды других проектов. Чтобы избежать несогласованности по целям и обеспечить эффективность использования ресурсов, необходимо интегрировать эти проекты в общую цифровую стратегию организации.

Помимо этого, необходимо обеспечить адекватную коммуникацию между заинтересованными сторонами. Разумным видится создание общей цифровой коммуникационной площадки в рамках компании, которая обладает функциями социальной сети и является доступной для всего персонала. Следует отметить, что в условиях самоизоляции компании для организации удаленной работы сотрудников создавали подобные коммуникационные площадки, в основном в рамках Zoom, Microsoft Teams и т.п. Однако эти площадки используются для конкретных мероприятий (совещаний, вебинаров и т.п.), не обеспечивая постоянную коммуникацию сотрудников, т.е. 
не обладают универсальной доступностью, какой обладает оффлайн-общение.

6. Прозрачность. В связи с тем, что организационные улучшения занимают достаточное количество времени, их успех реализуется в долгосрочной перспективе, они могут быть недоступны для понимания сотрудниками в краткосрочном периоде. Чтобы избежать недопонимания со стороны персонала и стимулировать эффективную работу, необходим план реализации цифровой трансформации, который связан с цифровой стратегией компании и доступен для каждого сотрудника. Каждый сотрудник должен понимать, что и зачем реализуется в настоящий момент в компании.

Успех цифровой трансформации напрямую зависит от готовности заинтересованных сторон к взаимодействию. Необходимо постоянно разъяснять сотрудникам преимущества, согласовывать соответствующие процедуры с организационными требованиями и обеспечивать достижение результатов.

Заключение. Несмотря на то, что цифровая трансформация является важной составляющей обеспечения конкурентоспособности компании, в настоящее время она является недостаточно изученной. Вместе с тем быстро изменяющаяся внешняя среда требует от компаний скорейших технологических изменений, адекватных требованиям времени. Иногда такие изменения происходят эволюционно, а иногда, как это произошло в условиях пандемии коронавируса, практически революционно, когда в кратчайшие сроки большинство компаний вынуждены были переводить сотрудников на удаленную работу.

Выделенные в данной статье рекомендации основаны на необходимости реализации ВРМ как основы эффективной цифровой трансформации.

Хотелось бы подчеркнуть, что цифровая трансформация - это не только процессы и технологии, это в большей степени люди, ее реализующие. Именно поэтому компаниям, прежде всего, необходимо уделять особое внимание обучению персонала, обеспечению доступности информации о целях и механизмах реализации стратегии цифровой трансформации, о характере принимаемых решений. Должна быть обеспечена эффективная коммуникация в рамках организации, должен быть сформирован «банк лучших практик» реализации процессов. Такой подход позволяет обеспечить вовлеченность каждого сотрудника в процесс реализации цифровой трансформации, а также обеспечить эффективность и результативность бизнес-процессов в организации.

\section{Литература}

1. Акмаева Р.И. Епифанова Н.Ш., Жуков В.M. Возможности адаптивной модели Agile для менеджмента // Вестник Астраханского государственного технического университета. Серия Экономика. - 2017. - №1. C. 7-15.

2. Ананьин В. И., Зимин К. В., Лугачев М.И., Гимранов Р.Д., Скрипкин К.Г. Цифровые предприятия: трансформация в новую реальность // Бизнес-информатика. — 2018. №2 (44). - C. 45-54.

3. Концепция Business Performance Мanagement: начало пути / Е.Ю. Духонин, Д.В. Исаев, Е.Л. Мостовой и др.; под ред. Г.В. Генса. - М.: Альпина Бизнес Букс, 2004. - 269 c.

4. Konn A.М., Орловский Д.Л. Подход к анализу и оптимизации моделей бизнеспроцессов в нотации BPMN // Радіоелектроніка, інформатика, управління. - 2018. №2. - C. 108-115.

5. Лыккова А.И., Батищев А.В. Развитие концепции управления бизнес-процессами организации: от классического подхода к ВРМ-системам // Синергия. - 2015. №1 . - C.48-54.

6. BharadwajA., El SawyO.A., Pavlou P.A., Venkatraman N.V. Visions and voices on emerging challenges in digital business strategy // MIS Quarterly. — 2013. — Vol.37. №2. - Pp. 633-661. - DOI:10.25300/ MISQ/2013/37.2.14.

7. Dürr S., Wagner H.-T., Weitzel T., Beimborn $D$. Navigating digital innovation: the complementary effect of organizational and knowledge recombination // 13th International Conference on Wirtschaftsinformatik. - St. Gallen, 2017 - Pp. 1363-1377.

8. Fischera M., Imgrunda F., Janiescha $C$., Winkelmanna A. Strategy archetypes for digital transformation: Defining meta objectives us- 
ing business process management [Electronic resource] // Information \& Management. 2020. - URL: https://doi.org/10.1016/j. im.2019.103262.

9. Grover $V$. The information systems field: making a case for maturity and contribution // Journal of the Association for Information Systems. - 2012. — №13 (4). - Pp. 254-272. DOI:10.17705/1jais.00291.

10. Hansen A. M., Kraemmergaard P., Mathiassen $L$. Rapid adaptation in digital transformation: a participatory process for engaging IS and business leaders // MIS Quarterly Executive. 2011. — №10 (4). - Pp. 175-185.

11. Hess T., Matt C., Benlian A., Wiesböck F. Options for Formulating a Digital Transformation Strategy // MIS Quarterly Executive. 2016. - Vol. 15. — №2. - Pp. 103-119.

12. Holotiuk F., Beimborn D. Critical success factors of digital business strategy // 13 International Conference on Wirtschaftsinformatik. St. Gallen, 2017. - Vol. 13. - Pp.991-1005.

13. Imgrund F., Fischer M., Janiesch $C$., Winkelmann A. Approaching Digitalization with Business Process Management [Electronic resource] // Multikonferenz Wirtschaftsinformatik (MKWI), 2018, Lüneburg. — Pp. 1725-1736. URL: https://pdfs.semanticscholar.org/1031/ f03b2f5a5f1686dd4d2a00f2d58d7510fc4d.pdf.

14. Santiago da Costa L., Pereira L., Santos Akkari A.C. Organizational Digital Transformation: A Proposed Instrument for Digitalization Management [Electronic resource] // Proceedings of the 4th Brazilian Technology Symposium (BTSym'18). - Pp. 419-426. URL: https://doi.org/10.1007/978-3-03016053-1_40.

15. Legner C., Eymann T., Hess T., Matt C., Böhmann T., Drews P., Mädche A., Urbach N., Ahlemann F. Digitalization: opportunity and challenge for the business and information systems engineering community // Business \& Information Systems Engineering. - 2017. №59 (4). - Pp. 301-308. - DOI:10.1007/ s12599-017-0484-2.

16. Matt C., Hess T., Benlian A. Digital transformation strategies // Business \& Information Systems Engineering. - 2015. №57(5). - Pp. 339-343. - DOI:10.1007/ s12599-015-0401-5.

17. Mithas $S$., Tafti A., Mitchell $W$. How a firm's competitive environment and digital stra- tegic posture influence digital business strategy // MIS Quarterly. — 2013. - Vol. 37. - Pp. 511536. - DOI:10.25300/MISQ/2013/37.2.09.

18. Rosemann M., J. vom Brocke. The six core elements of business process management / In J. vom Brocke, M. Rosemann (eds.) // Handbook on Business Process Management 1: Introduction, Methods and Information Systems. Springer, Berlin, 2015. - Pp. 105-122.

19. Singh A., Hess T. How chief digital officers promote the digital transformation of their companies // MIS Quarterly Executive. 2017. — №16 (1). — Pp. 1-17.

20. Zinder E., Yunatova I. Synergy for digital transformation: person's multiple roles and subject domains integration // In: Digital Transformation and Global Society. 2016. - Pp. 155-168.

\section{References}

1. Akmaeva R.I. Epifanova N. Sh., Zhukov V.M. Vozmozhnosti adaptivnoj modeli Agile dlja menedzhmenta [Possibilities of the adaptive Agile model for management] // Vestnik Astrahanskogo gosudarstvennogo tehnicheskogo universiteta. Serija Jekonomika [Bulletin of the Astrakhan State Technical University. Economy series]. - 2017. — №1. - Pp. 7-15.

2. Anan'in V. I., Zimin K. V., Lugachev M.I., Gimranov R.D., Skripkin K. G. Cifrovye predprijatija: transformacija v novuju real'nost' [Digital enterprises: transformation into a new reality]// Biznes-informatika. - 2018. - №2 (44). Pp. 45-54.

3. Koncepcija Business Performance Management: nachalo puti [The concept of Business Performance Management: the beginning of the path] / E. Ju. Duhonin, D. V. Isaev, E.L. Mostovoj i dr.; in G.V. Gens (eds.). - Moscow: Al'pina Biznes Buks, 2004. — 269 p.

4. Kopp A.M., Orlovskij D.L. Podhod k analizu i optimizacii modelej biznes-processov $\mathrm{v}$ notacii BPMN [An approach to the analysis and optimization of business process models in BPMN notation] // Radioelektronika, informatika, upravlinnja. - 2018. - №2. Pp. 108-115.

5. Lykova A.I., Batishhev A.V. Razvitie koncepcii upravlenija biznes-processami organizacii: ot klassicheskogo podhoda k BPM-sistemam [Development of the concept of management of 
business processes of the organization: from the classical approach to BPM systems] // Sinergija. - 2015. — №1. - Pp. 48-54.

6. Bharadwaj A., El SawyO.A., Pavlou P.A., Venkatraman N.V. Visions and voices on emerging challenges in digital business strategy // MIS Quarterly. - 2013. - Vol.37. №2. - Pp. 633-661. - DOI:10.25300/ MISQ/2013/37.2.14.

7. Dürr S., Wagner H.-T., Weitzel T., Beimborn $D$. Navigating digital innovation: the complementary effect of organizational and knowledge recombination // 13th International Conference on Wirtschaftsinformatik. - St. Gallen, 2017 - Pp. 1363-1377.

8. Fischera M., Imgrunda F., Janiescha C., Winkelmanna A. Strategy archetypes for digital transformation: Defining meta objectives using business process management [Electronic resource] // Information \& Management. 2020. - URL: https://doi.org/10.1016/j. im.2019.103262.

9. Grover $V$. The information systems field: making a case for maturity and contribution // Journal of the Association for Information Systems. - 2012. — №13 (4). — Pp. 254-272. DOI:10.17705/1jais.00291.

10. Hansen A. M., Kraemmergaard P., Mathiassen $L$. Rapid adaptation in digital transformation: a participatory process for engaging IS and business leaders // MIS Quarterly Executive. 2011. — №10 (4). — Pp. 175-185.

11. Hess T., Matt C., Benlian A., Wiesböck F. Options for Formulating a Digital Transformation Strategy // MIS Quarterly Executive. - 2016. - Vol. 15. - №2. - Pp. 103-119.

12. Holotiuk F., Beimborn D. Critical success factors of digital business strategy // 13 International Conference on Wirtschaftsinformatik. St. Gallen, 2017. - Vol. 13. - Pp.991-1005.

13. Imgrund F., Fischer M., Janiesch C., Winkelmann A. Approaching Digitalization with Business Process Management [Electronic resource] // Multikonferenz Wirtschaftsinformatik
(MKWI), 2018, Lüneburg. - Pp. 1725-1736. URL: https://pdfs.semanticscholar.org/1031/ f03b2f5a5f1686dd4d2a00f2d58d7510fc4d.pdf.

14. Santiago da Costa L., Pereira L., Santos Akkari A.C. Organizational Digital Transformation: A Proposed Instrument for Digitalization Management [Electronic resource] // Proceedings of the 4th Brazilian Technology Symposium (BTSym'18). - Pp. 419-426. URL: $\quad$ https://doi.org/10.1007/978-3-03016053-1 40.

15. Legner C., Eymann T., Hess T., Matt C., Böhmann T., Drews P., Mädche A., Urbach N., Ahlemann F. Digitalization: opportunity and challenge for the business and information systems engineering community // Business \& Information Systems Engineering. - 2017. №59 (4). - Pp. 301-308. - DOI:10.1007/ s12599-017-0484-2.

16. Matt C., Hess T., Benlian A. Digital transformation strategies // Business \& Information Systems Engineering. - 2015. №57(5). - Pp. 339-343. - DOI:10.1007/ s12599-015-0401-5.

17. Mithas S., Tafti A., Mitchell $W$. How a firm's competitive environment and digital strategic posture influence digital business strategy // MIS Quarterly. — 2013. - Vol. 37. — Pp. 511536. — DOI:10.25300/MISQ/2013/37.2.09.

18. Rosemann M., J. vom Brocke. The six core elements of business process management / In J. vom Brocke, M. Rosemann (eds.) // Handbook on Business Process Management 1: Introduction, Methods and Information Systems. Springer, Berlin, 2015. - Pp. 105-122.

19. Singh A., Hess T. How chief digital officers promote the digital transformation of their companies // MIS Quarterly Executive. 2017. — №16 (1). — Pp. 1-17.

20. Zinder E., Yunatova I. Synergy for digital transformation: person's multiple roles and subject domains integration // In: Digital Transformation and Global Society. 2016. - Pp. 155-168. 

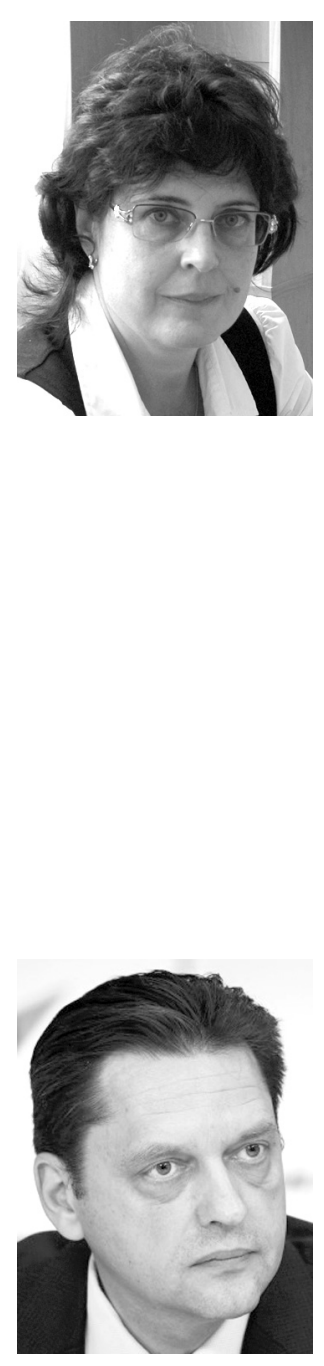

Овчинникова Оксана Петровна - доктор экономических наук, профессор, профессор кафедры прикладной экономики Центра управления отраслями промышленности экономического факультета Российского университета дружбы народов.

Ovchinnikova Oxana Petrovna - Doctor of Economic Sciences, Professor, Professor of the Department of Applied Economics, Center for Management of Industries, Faculty of Economics, Peoples' Friendship University of Russia.

117198 , г. Москва, ул. Миклухо-Маклая, 6 6 Miklukho-Maklaya st., 117198, Moscow, Russia

E-mail: ovchinnikova-op@rudn.ru

Харламов Максим Михайлович - кандидат экономических наук, первый заместитель начальника НИИ «Центр подготовки космонавтов им. Ю. А. Гагарина» по организации деятельности и инновационному развитию.

Kharlamov Maxim Mikhailovich - Candidate of Economic Sciences, first Deputy Head of the Yu. A. Gagarin Research \& Test Cosmonaut Training Center for the organization of activities and innovative development.

141160, Московская обл., Звездный городок

Star City, 141160, Moscow reg., Russia

E-mail:m.kharlamov@gctc.ru 